\title{
Facile and one-pot synthesis of uniform PtRu nanoparticles on polydopamine-modified multiwalled carbon nanotubes for direct methanol fuel cell application
}

\author{
Fengxia Chen, ${ }^{\text {a,b }}$ Junkai Ren, ${ }^{\mathrm{a}}$ Qian He,${ }^{\mathrm{a}}$ Jun Liu, ${ }^{\text {,* }}$ \\ Rui Song ${ }^{\mathrm{a}^{*}}$
}

${ }^{a}$ College of Chemistry and Chemical Engineering, University of Chinese Academy of Sciences, Beijing, 100049, China

${ }^{b}$ Program of Chemical and Biochemical Engineering, Sino-Danish College, Chinese Academy of Sciences, Beijing, 100190, China

${ }^{c}$ E.N.T, Renmin Hospical of Henan Province, Zhengzhou, 450000, China

Corresponding author: rsong@ucas.ac.cn (Rui Song)

*The first two authors contributed equally to this paper.

\begin{abstract}
A facile, environment-friendly and one-pot synthesis method for the preparation of high performance PtRu electrocatalysts on the multiwalled carbon nanotubes (MWCNTs) is reported. Herein, bimetallic PtRu electrocatalysts are deposited onto polydopamine (Pdop) -functionalized MWCNTs by mildly stirring at room temperature. Without the use of expensive chemicals or corrosive acids, this noncovalent functionalization of MWCNTs by Pdop is simple, facile and eco-friendly, and thus preserving the integrity and electronic structure of MWCNTs. Due to the well improved dispersion and the decreased size of alloy nanoparticles, the PtRu electrocatalysts on Pdop-functionalized MWCNTs show much better dispersion, higher electrochemically active surface area, and higher electrocatalytic activity for the electrooxidation of methanol in direct methanol fuel cells, compared with the conventional acid-treated MWCNTs.
\end{abstract}

Keywords: electrocatalyst, polydopamine, PtRu nanoparticles, one-pot synthesis, direct methanol fuel cell 


\section{Introduction}

The direct methanol fuel cells (DMFCs) have received considerable attentions for applications in transportation, portable electronics, and residential power sources, due to their high energy density, relatively low operating temperatures, zero or low emission of pollutants and minimal corrosion problems. However, the commercial viability of DMFCs is still hindered by several factors, including the low catalytic activity of electrodes both for the oxygen reduction reaction (ORR) and for the methanol oxidation reaction (MOR), the high costs of the Pt-based electrocatalysts, and the susceptibility of the catalysts to be poisoned by the CO-like intermediates formed in the methanol oxidation reaction. ${ }^{1,2}$

The most common solutions to these problems are to combine platinum with other metals such as $\mathrm{Ru}, \mathrm{Ni}, \mathrm{Co}$, etc. ${ }^{3-5}$, and meanwhile develop the morphology and distribution of the metals on proper supports. ${ }^{6-8}$ Over the last two decades, various Pt-based bimetallic catalysts have been studied. ${ }^{9-11}$ Among them, PtRu alloy catalyst is a leading candidate as a DMFCs anode since it shows high catalytic activity for methanol oxidation reaction as well as strong tolerance against carbon monoxide poisoning ${ }^{12-14}$, and in particular, the $\mathrm{Ru}$ in the PtRu alloy can easily oxidize the $\mathrm{CO}$ intermediate to $\mathrm{CO}_{2}$ by the bifunctional mechanism. ${ }^{15}$ Furthermore, these fabrication methods generally involve the reduction of metal ions using reducing agents such as hydrazine hydrate ${ }^{16}$, sodium borohydride ${ }^{17}$, formaldehyde ${ }^{18}$, ascorbic acid (AA) $)^{19,20}$ and dimethyl formamide ${ }^{21}$, etc. and most of these reductants are highly toxic and pose potential environmental and biological risks. Therefore, there is an urgent need to exploit eco-friendly, cost effective and highly active catalysts for methanol oxidation. ${ }^{22}$

On the other hand, as well realized that the proper selection of the carbon supporting materials for PtRu nanocatalyst would be helpful to overcome the problems such as slow kinetics of the oxidation reaction and poor tolerance to $\mathrm{CO}$ poisoning. Compared with smooth Pt and PtRu bulks, carbon-supported Pt and PtRu nanoparticles (NPs) show higher current efficiency for methanol oxidation due to their larger surface areas whereby the soluble intermediates formed are more readily adsorbed and oxidized to $\mathrm{CO}_{2}$ before 
they are transported away from the electrode surface by the continuous flow of electrolyte. ${ }^{23}$ In this regard, nano-carbons such as carbon nanoparticles, carbon nanotubes (CNTs), and graphene are thus used as the supporting materials for PtRu nanocatalyst in the anode of DMFC or DEFC. ${ }^{24}$

Comparatively, CNTs have attracted special attention as catalyst supports in fuel cell applications owing to their unique properties, such as large surface areas, high chemical resistibility, and superior mechanical strength. In addition, the hairlike structure of CNTs allows for entanglements among nanotubes, enabling better electron conductivity than traditional carbon systems. ${ }^{25}$ From this context, numerous studies have reported higher electrocatalytic activities of Pt-based NPs supported on CNTs as compared with those deposited on high-surface-area carbon. ${ }^{26,27}$ However, most of the established protocols to prepare CNTs-based electrocatalysts involve covalent functionalization of $\mathrm{CNTs}^{25,28,29}$ leading to the severe disruption of nanotubes' intrinsic electronic properties and thus increasing application expenditures. Other strategies consist of an adsorption of small organic coupling molecules ${ }^{30}$, polyelectrolytes ${ }^{10,11}$, polymers ${ }^{13}$ or surfactants ${ }^{31}$ to enable strong affinity of metal NPs on nanotubes. Nevertheless, such noncovalent modification introduces an issue of concern that the molecules absorbed on the surfaces of NPs may adversely affect their catalytic efficiencies.

Polydopamine (Pdop), which with a molecular structure similar to that of DOPA (3,4-dihydroxy-L-phenylalanine), moved into the spotlight as a novel coating material since 2007. ${ }^{32}$ One of Pdop's valuable features lies in its chemical structure that incorporates many functional groups such as catechol, amine and imine. These functional groups can serve as both the starting points for covalent modification with desired molecules and the anchors for the loading of transition or noble metal ions, which can further realize the emergence of diverse hybrid materials by virtue of its powerful reducing capability toward these metal ions under basic conditions. Liu et al. ${ }^{33}$ firstly reported that Pdop coatings contain abundant positively charged sites because of the nitrogen containing groups in the structure of Pdop, which enables Pdop to absorb on the surface of carbon materials through $\pi-\pi$ interaction and electrostatic interaction, forming 
electrostatic repulsion between carbon materials and thus preventing them from aggregating in aqueous solution. To the best of our knowledge, however, this Pdop-involved, noncovalent modification strategy has not been reported in the case of $\mathrm{PtRu}$ bimetallic catalysts in DMFC application.

Inspired by Liu's work, herein, we developed an efficient PtRu electrocatalyst, showing highly electrocatalytic activity for methanol oxidation and with excellent carbon monoxide $(\mathrm{CO})$ tolerance. As one part of significance in this work, we presented a facile and straightforward process to attach the PtRu nanoparticles (NPs) on the Pdop-modified MWCNTs (CNTs@Pdop). In this process no stabilizers (i.e., organic species, surfactants) for NPs and harsh pretreatments for MWNTs are required. The as-prepared CNTs@Pdop can form a stable suspension for several weeks in the absence of any stabilizers and accordingly the re-stacking of the CNTs is effectively inhibited, which thus supply ideal anchorage points for the PtRu NPs attachment; meanwhile, the chemical and crystallographic features, morphology, as well as electrocatalytic activity of the obtained catalysts were studied in detail via X-ray diffraction (XRD), X-ray photoelectron spectroscopy (XPS), transmission electron microscopy (TEM), etc. As indicated, the Pdop coating with an approximate several nanometers in thickness was uniformly deposited on MWCNTs, which leads to a homogenously distribution of PtRu NPs, and this consequently brings about significantly enhanced electrocatalytic activity for the methanol oxidation reaction (MOR).

\section{Experimental Section}

\section{Materials}

The multiwalled carbon nanotubes (MWCNTs, purity $=95 \%$, average diameter $=$ 10 20 nm, length $=5 \sim 15 \mu \mathrm{m}$ ), Shenzhen Nanotech Port Co. Ltd. Hexachloroplatinic (IV) acid hexahydrate $\left(\mathrm{H}_{2} \mathrm{PtCl}_{6} \cdot 6 \mathrm{H}_{2} \mathrm{O}\right)$, ruthenium trichloride $\left(\mathrm{RuCl}_{3}\right)$, Aladdin Reagent (Shanghai) Co., Ltd. 2-amino-2-hydroxymethylpropane-1,3-diol (tris), Sinopharm Chemical Reagent Co. Ltd. Dopamine hydrochloride, Alfa Aesar. All chemicals were of analytical grade and used as received. 


\section{Preparation of samples}

The purification of MWCNTs. Proper quantities of crude MWCNTs were added into a three-necked flask containing sulfuric acid and nitric acid in the ratio of 3:1 by volume. The suspension was sonicated in an ultrasonic bath for $40 \mathrm{~min}$ and then heated to $120{ }^{\circ} \mathrm{C}$ and refluxed for $3 \mathrm{~h}$. After centrifugation and later washing with hot distilled water until $\mathrm{pH} 7.0$, the purified MWCNTs were dried in a vacuum oven at $35{ }^{\circ} \mathrm{C}$ for $24 \mathrm{~h}$.

\section{Synthesis of Pdop coatings modified MWCNTs.}

The Pdop modified MWCNTs were synthesized according to the procedure described elsewhere. ${ }^{34}$ A typical experimental process is as follows: $20 \mathrm{mg}$ of MWCNTs were

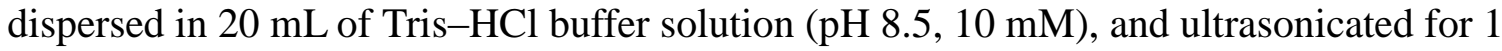
h. And then, $40 \mathrm{mg}$ of dopamine hydrochloride was added into solution and the mixture was stirred continuously for $24 \mathrm{~h}$ at ambient temperature. After centrifugation and washed three times with distilled water, the final products were dried in a vacuum oven at room temperature (labeled as CNTs@Pdop, hereinafter).

\section{Preparation of PtRu NPs/CNTs and PtRu/CNT@Dopa nanohybrids.}

The procedure for deposition of the PtRu NPs on the MWCNTs, i.e., PtRu/CNTs, was as follows: $20 \mathrm{mg}$ of CNTs was mixed with $438 \mu \mathrm{L} \mathrm{H}_{2} \mathrm{PtCl}_{6}(38.6 \mathrm{mM})$ in ethylene glycol. After ultrasonic treatment for $15 \mathrm{~min}, 350 \mu \mathrm{L} \mathrm{RuCl}_{3}(48.2 \mathrm{mM})$ was added into the above mixture, and further treated by ultrasonication for $30 \mathrm{~min}$. Subsequently, the $\mathrm{pH}$ value of the solution was adjusted to 8-9 with $1.0 \mathrm{M} \mathrm{KOH}$ aqueous solution, and the mixture was then treated with oil bath at $160{ }^{\circ} \mathrm{C}$ for $8 \mathrm{~h}$, followed by filtering, washing, and finally drying in vacuum oven at $60{ }^{\circ} \mathrm{C}$ for $12 \mathrm{~h}$. The PtRu electrocatalyst supported on the CNTs@Pdop was prepared in a similar fashion as described above with the CNTs replaced by the CNT @Dopa and was denoted as PtRu/CNTs@Pdop henceforth.

\section{Characterization}


Fourier-transform infrared spectra were recorded on TENSOR 27 FTIR spectrometer in transmittance mode with 32 scans at a resolution of $2 \mathrm{~cm}^{-1}$ interval. Thermogravimetric analysis (TGA) was performed on a PerkinElmer Diamond TA/TGA instrument. The samples were heated from 40 to $700{ }^{\circ} \mathrm{C}$ with a heating rate of $10{ }^{\circ} \mathrm{C}$ $\min ^{-1}$, and $\mathrm{N}_{2}$ flow of $50 \mathrm{~mL} \mathrm{~min}^{-1}$. Wide-angle X-ray diffraction (WAXD) was carried out using a Bruker D8 diffractometer with Ni-filtered $\mathrm{CuK} \alpha$ radiation $(\lambda=0.15418 \mathrm{~nm})$ operating at $40 \mathrm{kV}$ and $40 \mathrm{~mA}$. The XRD patterns were obtained at a scanning rate of $4^{\circ}$ $\min ^{-1}$ with an angular resolution of $0.05^{\circ}$ of the $2 \theta$ scan.

The average crystallite size of $\mathrm{Pt}$ particles was estimated from the peak of $\operatorname{Pt}(220)$ using the Scherrer's equation:

$$
\mathrm{d}=0.9 \lambda_{\mathrm{K} \alpha 1} / \beta \cdot \cos \theta_{\max }
$$

in which $d$ is the average size of the Pt particle, $\lambda_{\mathrm{K} \alpha 1}$ is the $\mathrm{X}$-ray wavelength $(\mathrm{Cu}, \mathrm{K} \alpha)=$ $0.15418 \mathrm{~nm}), \theta_{\max }$ is the maximum angle of the (220) peak, and $\beta$ is the full-width at half-maximum (FWHM) in radians.

Raman spectra were taken with Renishaw inVia-Raman Spectroscopy, equipped with a holographic grating of 1800 lines $\mathrm{mm}^{-1}$ and a HeNe laser $(632.8 \mathrm{~nm})$ as an excitation source. Specially adapted research grade 10X Leica microscope focused the excitation beam onto the samples.

Transmission electron microscopy (TEM, FEI Tecnai $\mathrm{G}^{2} 20$ ) experiments were conducted with an accelerating voltage of $120 \mathrm{kV}$. Prior to visualization, the specimen for TEM imaging was prepared from the suspension in deionized water under sonication for 5 min. Then, a drop of well-dispersed suspension was rightly placed on a micro grid and then drying in air.

X-ray photoelectron spectroscopy (XPS) data was recorded with an ESCA Lab220i-XL electron spectrometer using $300 \mathrm{~W}$ AlKa radiation, in which the binding energies were referenced to the $\mathrm{C} 1 \mathrm{~s}$ line at $284.8 \mathrm{eV}$ from adventitious carbon. The base pressure was about $3 \times 10^{-9}$ mbar. The source power was set to be $50 \mathrm{~W}$. The pass energies 
of $100 \mathrm{eV}$ for survey scans and $20 \mathrm{eV}$ for high resolution scans were used. The exact metal loading of the catalysts was accurately determined with an inductively coupled plasma atomic emission spectrometry (ICP-AES), Optima 8000, PerkinElmer Corp.

\section{Electrochemical measurements}

All electrochemical measurements were performed on a CHI 660E electrochemical workstation. A conventional three-electrode glass cell was used with a platinum wire as the counter electrode and a saturated calomel electrode (SCE) as the reference electrode. All the potentials reported herein were with respect to SCE. For electrochemical investigation, a glassy carbon (GC, $5 \mathrm{~mm}$ diameter) electrode was polished with the slurry of $1.5,0.5$ and $0.05 \mu \mathrm{m}$ alumina successively, and then washed ultrasonically in ethanol and pure water prior to use.

The catalyst inks were prepared as follows: $4 \mathrm{mg}$ of the materials dispersed in $1 \mathrm{ml}$ of 1:1 v/v water/isopropanol, and then $80 \mathrm{~mL} 5 \mathrm{wt} \%$ Nafion was added into the solution. The mixture was sonicated for more than 30 min to obtain a homogeneous ink. Then, 1 $\mathrm{mL}$ of the catalyst ink was loaded onto the GC electrode. Prior to oxygen reduction and methanol oxidation measurement, a cyclic voltammetry $(\mathrm{CV})$ profile was recorded in $\mathrm{N}_{2}$ saturated $0.5 \mathrm{M} \mathrm{H}_{2} \mathrm{SO}_{4}$ solution. Before the $\mathrm{CV}$ tests, $\mathrm{N}_{2}$ was bubbled through the electrolyte solution for $30 \mathrm{~min}$. The working electrodes were then electrochemically cleaned by sweeping the potential between $0.0 \mathrm{~V}$ and $1.4 \mathrm{~V}$ at $50 \mathrm{mV} \mathrm{s}^{-1}$ until steady-state $\mathrm{CVs}$ were obtained. Finally the potential was scanned in the potential range of $0.04 \mathrm{~V}$ to $1.24 \mathrm{~V}$ with a scan rate of $50 \mathrm{mV} \mathrm{s}^{-1}$ for 20 cycles. The stable $20^{\text {th }} \mathrm{CV}$ curve was used for calculating the electrochemical structure area (ECSA) of Pt component. The MOR measurements was conducted in the potential range between $0.04 \mathrm{~V}$ to $1.24 \mathrm{~V}$ in $\mathrm{H}_{2} \mathrm{SO}_{4}$ and $1 \mathrm{M} \mathrm{CH}_{3} \mathrm{OH}$.

\section{Results and Discussions}

Fig. 1 illustrates the synthesis route of the PtRu NPs immobilization on the nano-shell Pdop-coating modified MWCNTs. The chemical structure comprising both alkylamine and catechol functionalities enables dopamine to undergo self-polymerization and 
changes into Pdop in the conditions of slightly alkaline solution and the presence of air. ${ }^{32}$ The MWCNTs functionalized with nanoshell Pdop coating have a large amount of positively charged sites because of the nitrogen-containing groups in the structure of Pdop. ${ }^{35}$ In this case, the metal-binding ability of nitrogen-containing groups and hydroxyl (-OH) present in the Pdop layer was used to absorb $\mathrm{PtCl}_{6}{ }^{2-}$ and $\mathrm{Ru}^{3+}$ ions onto the carbon materials surface ${ }^{36}$, and additionally, the weak reducibility of Pdop was utilized to reduce the absorbed ions into Pt or Ru NPs immobilized onto carbon materials surface.

The weight percentage of surface-functionalized Pdop moieties on MWCNTs is evaluated by TGA analysis. For the acid oxidation-treated MWCNTs, the weight loss in the temperature of $100-700{ }^{\circ} \mathrm{C}$ range is ca. $11.42 \mathrm{wt} \%$, which mainly attributed to $-\mathrm{OH}$, $-\mathrm{COOH}$ and the amorphous carbon particles. However, for Pdop-modified MWCNTs, the corresponding weight loss should be ascribed mainly to the pyrolysis of Pdop moiety on the surface of MWCNTs (data was not shown). Thus, from the mass loss in TGA curves of the neat Pdop and the MWCNTs, the mass ratio of Pdop layer against the encircled MWCNTs is estimated to be ca. $4.93 \mathrm{wt} \%$.

The purified MWCNTs, Pdop and CNTs@Pdop were characterized by FTIR and Raman spectroscopy (Fig. 2). Dopamine shows many narrow peaks, a feature of small molecule; while Pdop presents only a few intense absorption features: $\sim 1615 \mathrm{~cm}^{-1}$ from aromatic rings and $\sim 3420 \mathrm{~cm}^{-1}$ from catechol $-\mathrm{OH}$ groups (Fig. 2A). The pure MWCNTs show weak absorption at the same positions: $\sim 1615$ and $\sim 3420 \mathrm{~cm}^{-1}$, implying the presence of acid group on MWCNTs surface, and that the MWCNTs structure has affinity to Pdop aromatic rings. Comparatively, the prepared CNTs@Pdop shows mainly the absorption features of Pdop component. It is noteworthy that, for CNTs@Pdop, the retention of peaks indicative of the neat MWCNTs after the Pdop deposition suggests the undisturbed MWCNTs structure and the wrapping of MWCNTs by Pdop as well. Furthermore, as seen in Raman spectra in Fig. 2B, the purified MWCNTs depict its featured $\mathrm{G}$ and $\mathrm{D}$ bands at $\sim 1570$ and $\sim 1350 \mathrm{~cm}^{-1}$, due to the graphene structure with some defects. While, the Pdop layer presents broad peaks at the same locations: $\sim 1570$ and $\sim 1350 \mathrm{~cm}^{-1}$. Collectively, the CNTs@Pdop reveals the sum features of MWCNTs 
and Pdop, and the similarity in FTIR and Raman spectra support the affinity between the MWCNTs and Pdop layer. ${ }^{34}$

An ideal dispersion of the PtRu catalyst on the carbon support is a precondition for attaining good electrocatalytic activity in a fuel cell reaction. Roughly yet clearly, the Pdop modification could profoundly enhance the MWCNTs dispersity and its stability in water, in which the sonicated CNTs@Pdop remained well-dispersed ever after 2 week settlement at ambient condition (the insets of Fig. 2A), and this could be further visualized from the TEM images in Fig. 3B1, in which the Pdop coating features as a thin amorphous-like layer with a thickness of ca. 2-7 nm around the CNT surface (Fig. 3). In addition, TEM images of PtRu NPs on CNTs@Pdop, i.e., PtRu/CNTs@Pdop are shown in Fig.3B2 and Fig. 3B3, in low and high magnification, respectively. Comparing with the MWCNTs support, the PtRu NPs are more homogeneously decorated on the outer surface of CNTs@Pdop. A statistical size distribution obtained from 200 randomly selected NPs on CNTs@Pdop support shows that the size distribution of the PtRu NPs is quite narrow with an average diameter of $\sim 2.3 \mathrm{~nm}(v s . \sim 3.6 \mathrm{~nm}$ for PtRu NPs on acid-purified CNTs).

Fig. 4 shows the XRD patterns of MWCNTs, PtRu/CNTs and PtRu/CNTs@Pdop. As indicated, distinct diffraction peaks ca. $26^{\circ}(2 \theta)$ of the hexagonal structure of graphitic carbon in neat MWCNTs is observed (JCPDS, No. 75-1621). ${ }^{37}$ Moreover, the diffraction peaks at $\sim 39.6^{\circ}, \sim 46.3^{\circ}$ and $\sim 67.4^{\circ}$, which being assigned respectively to $\operatorname{Pt}(111), \operatorname{Pt}(200)$ and $\operatorname{Pt}(220)$, could be seen. However, the diffraction peaks near $38^{\circ}$ and $44^{\circ}$ in $2 \theta$ from $\mathrm{Ru}$, are not observed, and this observation suggest that Ru may enter the Pt lattice to form the PtRu alloy or Ru exists in the amorphous state. ${ }^{38}$ One noteworthy issue is that, the $\operatorname{Pt}(220)$ 
diffraction peak at $\sim 67.4^{\circ}$ are broader and weaker for PtRu/CNTs@ Dopa than that for PtRu/CNTs, indicating the smaller size of PtRu NPs on CNTs@Pdop. In this case, the $\operatorname{Pt}(220)$ band, instead of the $\operatorname{Pt}(111)$ band, was chosen for fitting, in that this band was not subject to interference from other peaks and therefore can provide better accuracy. On the basis of Scherrer's equation through line broadening of the $\operatorname{Pt}(220)$ peak (eq. 1), the average size of Pt NPs for PtRu/CNTs@Pdop and PtRu/CNTs was calculated to be $\sim 2.5$ and $\sim 3.8 \mathrm{~nm}$, respectively, and these values are quite consistent with the above TEM results.

XPS analyses are performed to investigate the chemical nature of PtRu/CNTs@Pdop (Fig. 5). Besides the $\mathrm{C} 1 \mathrm{~s}$ signal at $284.8 \mathrm{eV}$, the O1s, Pt4f, N1s, Ru3p and $\mathrm{Pt}_{4 \mathrm{~d}}$ signals appear in all samples. As shown in Fig. 5a, the peak at $398.7 \mathrm{eV}$ demonstrates the presence of $\mathrm{Pt}-\mathrm{N}$ or $\mathrm{Ru}-\mathrm{N}$ bonding between the $\mathrm{N}$ atoms of Pdop and the surface $\mathrm{Pt}$ or $\mathrm{Ru}$ atoms of the PtRu NPs. These spectral results illustrate the bifunctional stabilizing mechanism of Pdop between PtRu NPs and CNTs.

The Pt4f line of PtRu/CNTs@Pdop in Fig. 5b shows a doublet containing a low-energy band $\left(\mathrm{Pt}_{4} \mathrm{f}_{7 / 2}\right)$ and a high-energy band $\left(\mathrm{Pt}_{4} \mathrm{f}_{5 / 2}\right)$ at $\sim 71.65$ and $\sim 74.95 \mathrm{eV}$, respectively. Whereas, the peaks for Pt $2 \mathrm{t}$ and $\mathrm{Pt} 4 \mathrm{t}$ at $\sim 73.8 \mathrm{eV}$ and $\sim 74.6 \mathrm{eV}$, respectively, are not found, indicating that $\mathrm{Pt}$ is present in the zero-valent metallic state in the nanoparticles. Further, in order to identify different oxidation states of Pt, the spectrum was deconvoluted into two pairs of peaks at $\sim 71.57 \mathrm{eV}, \sim 72.38 \mathrm{eV}$ and $\sim 74.91 \mathrm{eV}$, $\sim 76.09 \mathrm{eV}$. The more intense doublet peaks with binding energies of $\sim 71.56 \mathrm{eV}\left(\mathrm{Pt}_{4} \mathrm{f}_{7 / 2}\right)$ and $\sim 74.90 \mathrm{eV}\left(\mathrm{Pt}_{4} \mathrm{f}_{5 / 2}\right)$ are attributed to the metallic $\mathrm{Pt}$, while the second deconvoluted doublets observed at higher binding energy than $\mathrm{Pt}^{0}$ at $\sim 72.38 \mathrm{eV}$ and $\sim 76.09 \mathrm{eV}$ can be assigned to $\mathrm{Pt}^{2+}$ in $\mathrm{PtO}$ and $\mathrm{Pt}(\mathrm{OH})_{2}$-like species. ${ }^{39}$

It should be noted that the $\mathrm{Ru}$ oxide is a mixed electron/proton conductor, and is especially beneficial for methanol oxidation on $\mathrm{PtRu} .{ }^{40}$ In this case, $\mathrm{Ru} 3 \mathrm{p}_{3 / 2}$ is used for the surface analysis instead of that $\mathrm{Ru} 3 \mathrm{~d}_{3 / 2}$ peak at $\sim 280 \mathrm{eV}$ as the latter overlaps the $\mathrm{C} 1 \mathrm{~s}$ 
peak in the region of $\sim 280-290 \mathrm{eV}$. Accordingly, the $\mathrm{Ru} 3 \mathrm{p}_{3 / 2}$ peak is deconvoluted considering the contribution of $\mathrm{Ru}$ at $\sim 460.21 \mathrm{eV}$, and $\mathrm{RuO}_{2}$ at $\sim 461.11 \mathrm{eV}$ (Fig. $5 \mathrm{c}$ ).

Cyclic voltammetry (CV) curves of three catalysts were recorded in $0.5 \mathrm{M}$ $\mathrm{N}_{2}$-saturated $\mathrm{H}_{2} \mathrm{SO}_{4}$ solution with a sweep rate of $50 \mathrm{mV} \mathrm{s}^{-1}$ (Fig. 6A). Compared to other two catalysts, PtRu/CNTs@Pdop catalyst exhibits more distinct chemical hydrogen absorption and desorption peaks $(-0.3-0 \mathrm{~V})$, which might be due to the isotropy of individual PtRu nanocluster.

Moreover, the electrochemically active surface area (ECSA), corresponding to the number of available active site, was estimated by integrating the charge passed during the hydrogen adsorption/desorption as the following equation:

$$
\mathrm{ECSA}=\frac{\mathrm{Q}_{\mathrm{co}}}{\left[\mathrm{P}_{\mathrm{t}} \times 420\right] \mu \mathrm{C} \mathrm{cm}{ }^{-2}}
$$

where $\mathrm{Q}_{\mathrm{co}}$ is the charge for the $\mathrm{CO}$ stripping $\left(\mu \mathrm{C} \mathrm{cm}^{-2}\right),[\mathrm{Pt}]$ is the platinum loading $(\mathrm{mg}$ $\mathrm{cm}^{-2}$ ) in the electrode, and $420 \mu \mathrm{C} \mathrm{cm}^{-2}$ represents the charge density which is required to oxidize a monolayer of $\mathrm{CO}$ on the Pt site. It should be noted that this equation is based on the assumption that the charge required to oxide a monolayer of hydrogen on Pt surface is $210 \mu \mathrm{C} \mathrm{cm}^{-2}{ }^{39}$ In this study, the calculated ECSA values were $\sim 41.87 \mathrm{~m}^{2} \mathrm{~g}^{-1}, \sim 28.81$ $\mathrm{m}^{2} \mathrm{~g}^{-1}$ for PtRu/CNTs@Pdop and PtRu/CNTs, respectively. The much higher ECSA of PtRu/CNTs@Pdop is most likely due to, as depicted in the TEM images (Fig. 3A and $3 \mathrm{~B})$, its smaller PtRu particle size $(\sim 2.3 \mathrm{~nm})$ compared with that of the case in PtRu/CNTs ( $3.6 \mathrm{~nm}$ ), leading to higher exposure of surface even with the same PtRu loading.

Fig. $6 \mathrm{~b}$ shows the cyclic voltammograms for methanol oxidation in a solution of $0.5 \mathrm{M}$ $\mathrm{H}_{2} \mathrm{SO}_{4}+1 \mathrm{M} \mathrm{CH}_{3} \mathrm{OH}$. For the methanol oxidation, PtRu catalysts deposited on CNTs or CNTs@Pdop showed similar trends. The addition of methanol to the electrolyte results in a dramatic change in the appearance of voltammograms due to MOR taking place on the surface of the nanocatalysts. Methanol oxidation was characterized by the well-separated anodic peaks in the forward $\left(\mathrm{I}_{\mathrm{f}}\right)$ and reverse $\left(\mathrm{I}_{\mathrm{b}}\right)$ scans. Principally, the magnitude of peak 
in forward scan was directly proportional to the amount of methanol oxidized at the nanocatalyst electrode, while the reverse scan was attributed primarily to the removal of carbonaceous species not completely oxidized during the forward scan, such as $\mathrm{CO}$, which accumulated on the catalyst surface during the forward scan and thus decrease the electrocatalytic performance of the catalyst. ${ }^{41}$

Therefore, the peak current density of the forward scan $\left(\mathrm{I}_{\mathrm{f}}\right)$ is usually used to assess the electrocatalytic activity of a catalyst for methanol oxidation, that is, the higher the peak current density of methanol oxidation in the forward scan implies the better electrocatalytic activity of the catalysts. On the other hand, the ratio $\left(\mathrm{I}_{\mathrm{f}} / \mathrm{I}_{\mathrm{b}}\right)$ of peak currents associated with the anodic peaks in forward and reverse was normally used to describe the tolerance of a catalyst to intermediates generated during the oxidation of methanol. ${ }^{42}$ A higher $I_{f} / I_{b}$ value implies a better tolerance to $\mathrm{CO}$ and more complete oxidation of methanol to $\mathrm{CO}_{2}$ in the forward scan. ${ }^{43}$ The $\mathrm{I}_{\mathrm{f}} / \mathrm{I}_{\mathrm{b}}$ ratio $(\sim 1.2)$ of PtRu/CNTs@Pdop, is higher than that ( 0.8) of PtRu/CNTs, implying a relatively better poison tolerance of PtRu/CNTs@Pdop. The higher catalytic activity of PtRu/CNTs@Pdop comparing with PtRu/CNTs could be attributed to the presence of Pdop in the former catalyst, which could improve the stability of PtRu NPs and favor CO oxidation at lower potential than that on naked CNTs support. ${ }^{44}$

Stability is an important criterion in the development of electrocatalysts. After 1000 cycles, the current density value of the PtRu/CNTs@Pdop remained considerably higher than that of the PtRu/CNTs (Fig. 6c). Relative to the maximum current density value ( 42.4 mA cm ${ }^{-2}$ for the PtRu/CNTs@Pdop; $38.2 \mathrm{~mA} \mathrm{~cm}^{-2}$ for PtRu/CNTs), the loss of activity of the PtRu/CNTs@Pdop and the PtRu/CNTs is $\sim 13.2 \%$ and $\sim 28.9 \%$, respectively. The reason for the improved durability of the PtRu/CNTs@Pdop may be attributed to the ultrafine grain size of PtRu bimetallic nanoparticles, the synergetic effect of the $\mathrm{Pt}$ and $\mathrm{Ru}$ atoms, as well as the stick-and-coordinated effect of the biomimic dopamine moiety, which has been reported in the MOR with PdPt bimetallic nanoalloys as electrocatalysts. ${ }^{45}$ 


\section{Conclusions}

In summary, a facile, one-pot synthesis method for the preparation of high performance PtRu electrocatalysts on the MWCNTs is reported. Herein, bimetallic PtRu electrocatalysts are deposited onto polydopamine (Pdop) - functionalized MWCNTs by mildly stirring at room temperature. Without the use of expensive chemicals or corrosive acids, this noncovalent functionalization of MWCNTs by Pdop is remarkably simple and facile, and thus preserving the integrity and electronic structure of MWCNTs. Due to the well improved dispersion and the decreased size of alloy nanoparticles, the ultrafine size PtRu electrocatalysts on Pdop-functionalized MWCNTs show higher electrochemically active surface area, higher electrocatalytic activity as well as enhanced catalytic stability for the electrooxidation of methanol in direct methanol fuel cells, compared with the conventional acid-treated MWCNTs. This fabricating protocol may find useful roles in catalysis, biosensors, energy storage systems, and the even expanding other technological applications.

Acknowledgements This work was supported by the National Natural Science Foundation of China (21072221, 21172252). 


\section{References}

[1] X.S. Zhao, W. Li, Y.Z. Fu, and A. Manthiram, Intertional Journal Hydrogen Energy, 37(12), 9845 (2012).

[2] B. Bennett, B.M. Koraishy, and J.P. Meyers, Journal of Power Sources, 218(1), 268 (2012).

[3] S. Shahrokhian, and S. Rastgar, Electrochimica Acta, 58, 125 (2011).

[4] Y. Zhao, Y.F. E, L.Z. Fan, Y.F. Qiu, and S.H. Yang, Electrochimica Acta, 52, 5873 (2007).

[5] H.F. Cui, J.S. Ye, X. Liu, W.D. Zhang, and F.S. Sheu, Nanotechnology, 17, 2334 (2006).

[6] C. Nethravathi, E.A. Anumol, M. Rajamathi, and N. Ravishankar, Nanoscale, 3, 569 (2011).

[7] D. He, L. Yang, S. Kuang, and Q. Cai, Electrochemical Communication, 9, 2467 (2007).

[8] F.J. Rodriguez-Nieto, T.Y. Morante-Catacora, and C.R. Cabrera, Journal Electroanalysis Chemistry, 571, 15 (2004).

[9] N. Kakati, S.H. Lee, J. Maiti, and Y.S. Yoon, Surface Science, 606, 1633 (2012).

[10] R. Escudero-Cid, P. Hernandez-Fernandez, J.C. Perez-Flores, S. Rojas, S. Garcia-Rodriguez, E. Fatas, and P. Ocon, International Journal Hydrogen Energy, 37(13), 7119 (2012).

[11] S. Kang, S. Lim, D.H. Peck, S.K. Kim, D.H. Jung, S.H. Hong, H.G. Jung, and Y. Shul, International Journal Hydrogen Energy, 37(7), 4685 (2012).

[12] R. Basnayake, Z.R. Li, S. Katar, W. Zhou, H. Rivera, E.S. Smotkin, D.J. Casadonte, and C. Korzeniewski, Langmuir, 22(25), 10446 (2006).

[13] S.H. Ahn, I. Choi, O.J. Kwon, and J.J. Kim, Chemical Engineering Journal, 181, 276 (2012).

[14] B. Li, D.C. Higgins, S.M. Zhu, H. Li, H.J. Wang, J.X. Ma, and Z.W. Chen, Catalysis Communication, 18(1), 51 (2012).

[15] W.Z. Li, X. Wang, Z.W. Chen, M. Waje, and Y.S. Yan, Journal Physics Chemistry B, 110 (17), 15353 (2006).

[16] S.J. Guo, S.J. Dong, and E.K. Wang, ACS Nano, 4(1), 547 (2010).

[17] S.J. Kwon, and A.J. Bard, Journal of the American Chemical Society, 134(16), 10777 (2012).

[18] J.P. Wang, P. Holt-Hindle, D. MacDonald, D.F. Thomas, and A.C. Chen, Electrochimica Acta, 53, 6944 (2008).

[19] L. Wang, Y. Nemoto, and Y. Yamauchi, Journal of the American Chemical Society, 133(17), 9674 (2011).

[20] L. Wang, and Y. Yamauchi, Chemistry-European Journal, 17(14), 8810 (2011).

[21] S.J. Guo, and S.H. Sun, Journal of the American Chemical Society, 134(4), 2492 (2012). 
[22] Z.B. Wang, P.J. Zuo, G.J. Wang, C.Y. Du, and G.P. Yin, Journal Physics Chemistry C, 112(7), 6582 (2008).

[23] H.S. Wang, L.R. Alden, F.J. DiSalvo, and H.D. Abruna, Langmuir, 25(12), 7725 (2009).

[24] H.J. Huang, and X. Wang, Journal of Material Chemistry A, 2(11), 6266 (2014)..

[25] Y.L. Hsin, K.C. Hwang, and C.T. Yeh, Journal of the American Chemical Society, 129(16), 9999 (2007).

[26] M. Okamoto, T. Fujigaya, and N. Nakashima, Small, 5(2), 735 (2009).

[27] B.H. Wu, D. Hu, Y.J. Kuang, B. Liu, X.H. Zhang, and J.H. Chen, Angewdandte Chemie-Internaitonal Edition, 48(9), 4751 (2009).

[28] R. Chetty, W. Xia, S. Kundu, M. Bron, T. Reinecke, W. Schuhmann, and M. Muhler, Langmuir, 25(7), 3853 (2009).

[29] C.M. Zhou, H.J. Wang, F. Peng, J.H. Liang, H. Yu, and J. Yang, Langmuir, 25(12), 7711 (2009).

[30] D.Q. Yang, B. Hennequin, and E. Sacher, Chemistry of Material, 18(9), 5033 (2006).

[31] C.L. Lee, Y.C. Ju, P.T. Chou, Y.C. Huang, L.C. Kuo, and J.C. Oung, Electrochemical Communication, 7, 453 (2005).

[32] H. Lee, S.M. Dellatore, W.M. Miller, and P.B. Messersmith, Science, 318, 426 (2007).

[33] X.C. Liu, G.C. Wang, R.P. Liang, L. Shi, and J.D. Qiu, Journal of Material Chemistry A, 1(6), 3945 (2013).

[34] B. Fei, B.T. Qian, Z.Y. Yang, R.H. Wang, W.C. Liu, C.L. Mak, and J.H. Xin, Carbon, 46(4), 1795 (2008).

[35] B. Yue, Y.W. Ma, H.S. Tao, L.S. Yu, G.Q. Jian, X.Z. Wang, X.S. Wang, Y.N. Lu, and Z. Hu, Journal of Material Chemistry, 18(3), 1747 (2008).

[36] S.J. Jiang, Y.W. Ma, G.Q. Jian, H.S. Tao, X.Z. Wang, Y.N. Fan, Y.N. Lu, Z. Hu, and Y. Chen, Advcanced Material, 21(11), 4953 (2009).

[37] D. Sebastian, A.G. Ruiz, I. Suelves, R. Moliner, M.J. Lazaro, V. Baglio, A. Stassi, and A.S. Arico, Applied Catalysis B-Environmental, 115(1), 269 (2012).

[38] J.T. Moore, J.D. Corn, D. Chu, R.Z. Jiang, D.L. Boxall, E.A. Kenik, and C.M. Lukehart, Chemistry of Material, 15(6), 3320 (2003).

[39] R. Chetty, S. Kundu, W. Xia, M. Bron, W. Schuhmann, V. Chirila, W. Brandl, T. Reinecke, and M. Muhler, Electrochimica Acta, 54, 4208 (2009).

[40] Y.Z. Lu, and W. Chen, Chemical Communications, 47(5), 2541 (2011).

[41] C.S. Chen, F.M. Pan, and H.J. Yu, Applied Catalysis B-Environmental, 104(1), 382 (2011).

[42] M. Watanabe, and S. Motoo, Journal of Electroanalytical Chemistry, 60, 275 (1975).

[43] F.H. Li, Y.Q. Guo, T. Wu, Y. Liu, W. Wang, and J.P. Gao, Electrochimica Acta, 111, 614 (2013). 
[44] F.F. Ren, C.Y. Zhai, M.S. Zhu, C.Q. Wang, H.W. Wang, D. Bin, J. Guo, P. Yang, and Y.K. Du, Electrochimica Acta, 153, 175 (2015).

[45] Y. Liu, M.F. Chi, V. Mazumder, K.L. More, S. Soled, J.D. Henao, and S.H. Sun, Chemistry of Material, 23(7), 4199 (2011).

\section{Figure captions}

Figure 1. Schematic route for the preparation of the PtRu NPs immobilized on the Pdop coating -modified MWCNTs, i.e., CNTs@Pdop.

Figure 2. FTIR (A) and Raman spectra (B) of MWCNTs, Pdop and CNTs@Pdop. The insets in (A) comparatively shown the sedmetation of MWCNTs and CNTs@Pdop subject to sonication process, (a) as resultant; (b) after settled at ambient condition for 2 weeks.

Figure 3. TEM images of acid-purified MWCNTs (A1) and CNTs@Pdop (B1), in which the white line indicated the approximate thickness of Pdop layer. TEM images and size distribution histograms of PtRu/CNTs (A2, A3 and A4) and PtRu/CNTs@Pdop (B2, B3 and B4). The PtRu loading was ca. 20 wt\% assessed by ICP-AES.

Figure 4. XRD patterns of purified MWCNTs, PtRu/CNTs and PtRu/CNTs@Pdop. The PtRu loading was ca. $20 \mathrm{wt} \%$.

Figure 5. XPS survey spectrum of PtRu/CNTs@Pdop (A), high resolution XPS spectrum of $\mathrm{Pt}_{4 \mathrm{f}}(\mathrm{B})$, $\mathrm{Ru}_{3 \mathrm{p} 3 / 2}(\mathrm{C})$, and C $1 \mathrm{~s}+\mathrm{Ru} 3 \mathrm{~d}(\mathrm{D})$.

Figure 6. Cyclic voltammograms for the PtRu/CNTs@Pdop, PtRu/CNTs and CNTs@Pdop (Pt NPs loading $\sim 20 \mathrm{wt} \%$, assed by ICP-AES) recorded at room temperature in an $\mathrm{N}_{2}$-saturated $0.5 \mathrm{M} \mathrm{H}_{2} \mathrm{SO}_{4}$ solution at a scan rate of $50 \mathrm{mV} \mathrm{s}^{-1}$ (A). Cyclic voltammograms for methanol oxidation on the PtRu /CNTs@Pdop, PtRu/CNTs and CNTs@Pdop recorded at room temperature in a $0.5 \mathrm{M} \mathrm{H}_{2} \mathrm{SO}_{4}+1.0 \mathrm{M}$ methanol solution at a scan rate of $50 \mathrm{mV} \mathrm{s}^{-1}(\mathrm{~B})$. Forward peak current densities for methanol oxidation as a function of scanning cycles in a $0.5 \mathrm{M} \mathrm{H}_{2} \mathrm{SO}_{4}+1.0 \mathrm{M}$ methanol solution at a scan rate of $50 \mathrm{mV} \mathrm{s}^{-1}$ (C). 


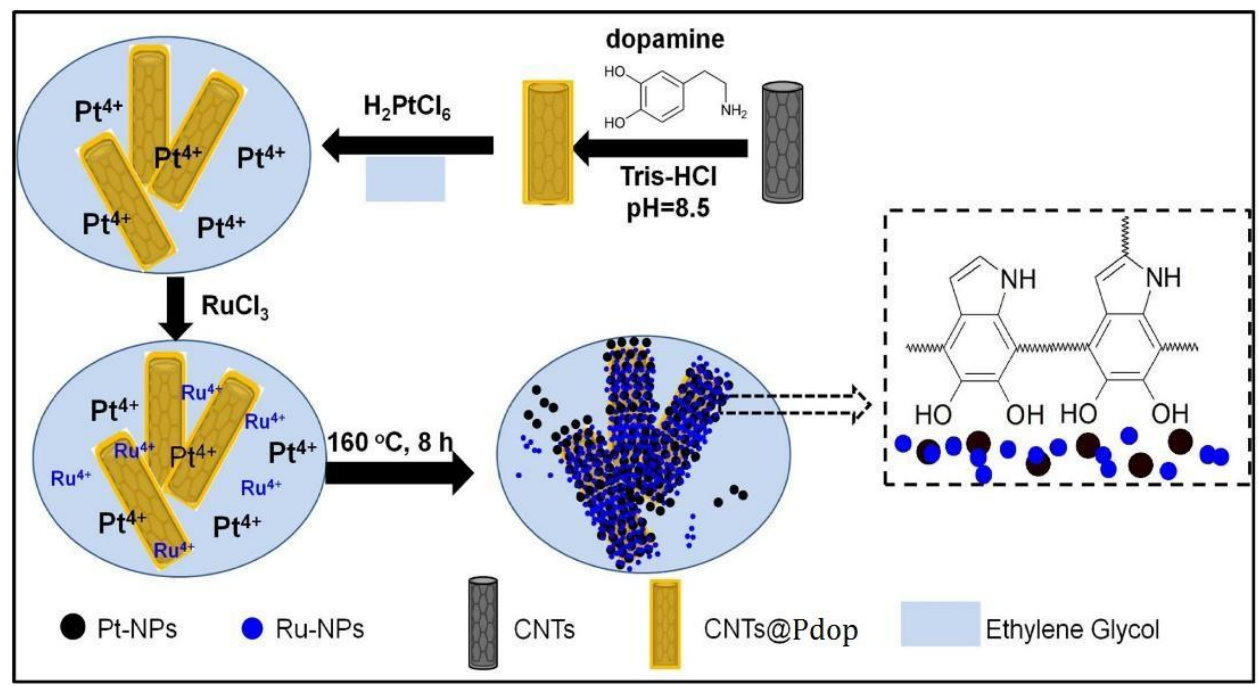

Figure 1. 

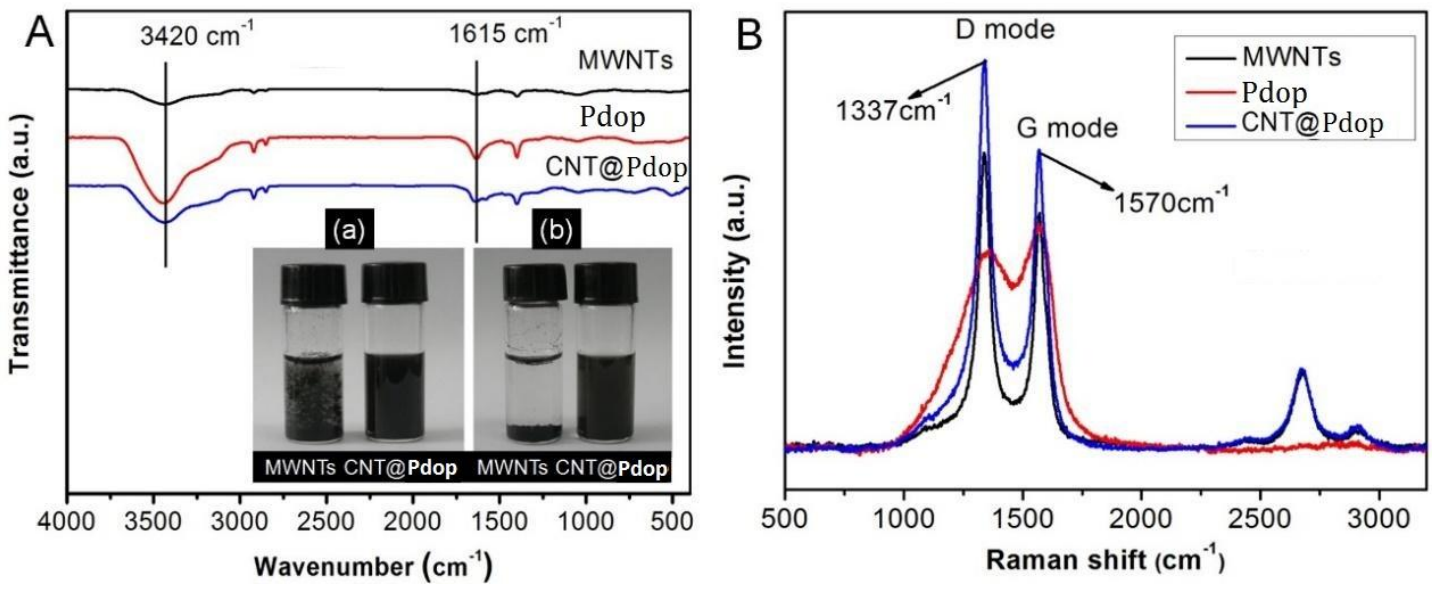

Figure 2. 

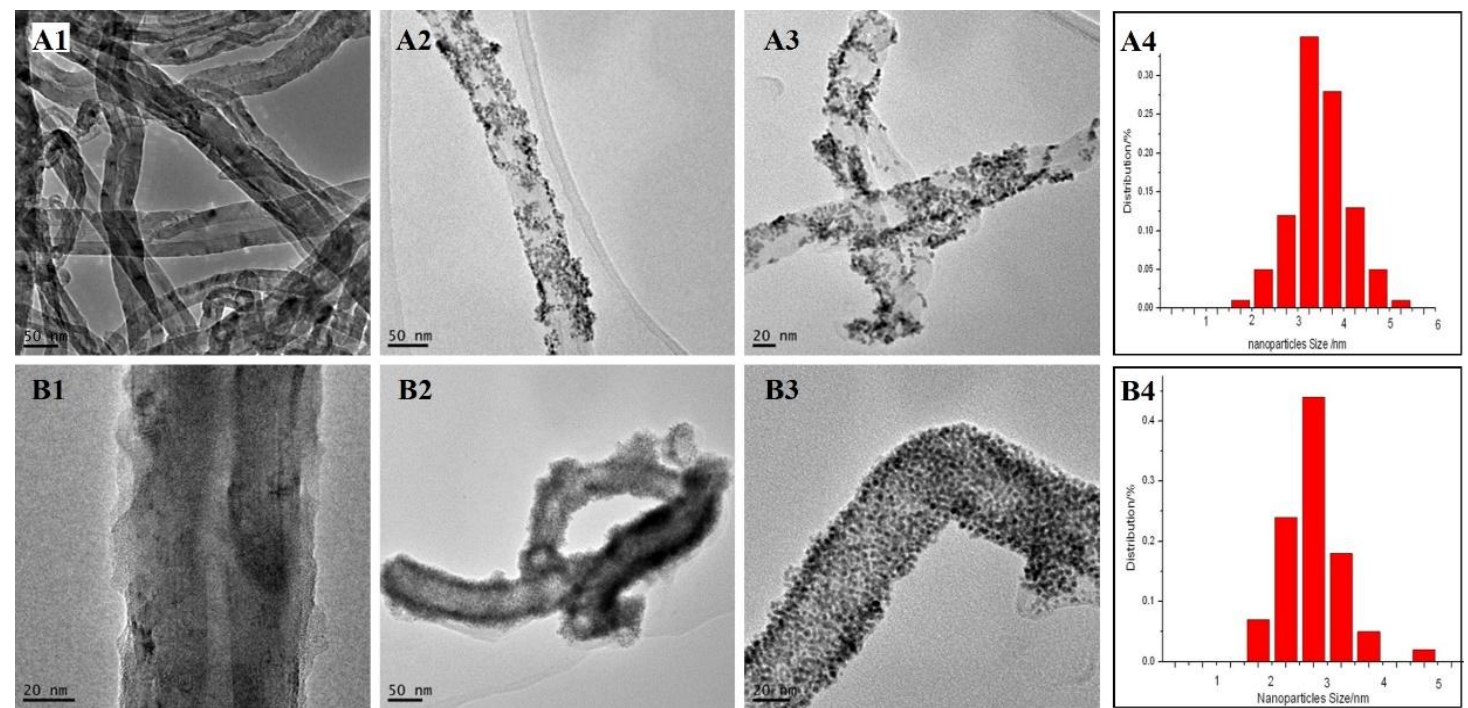

Figure 3. 


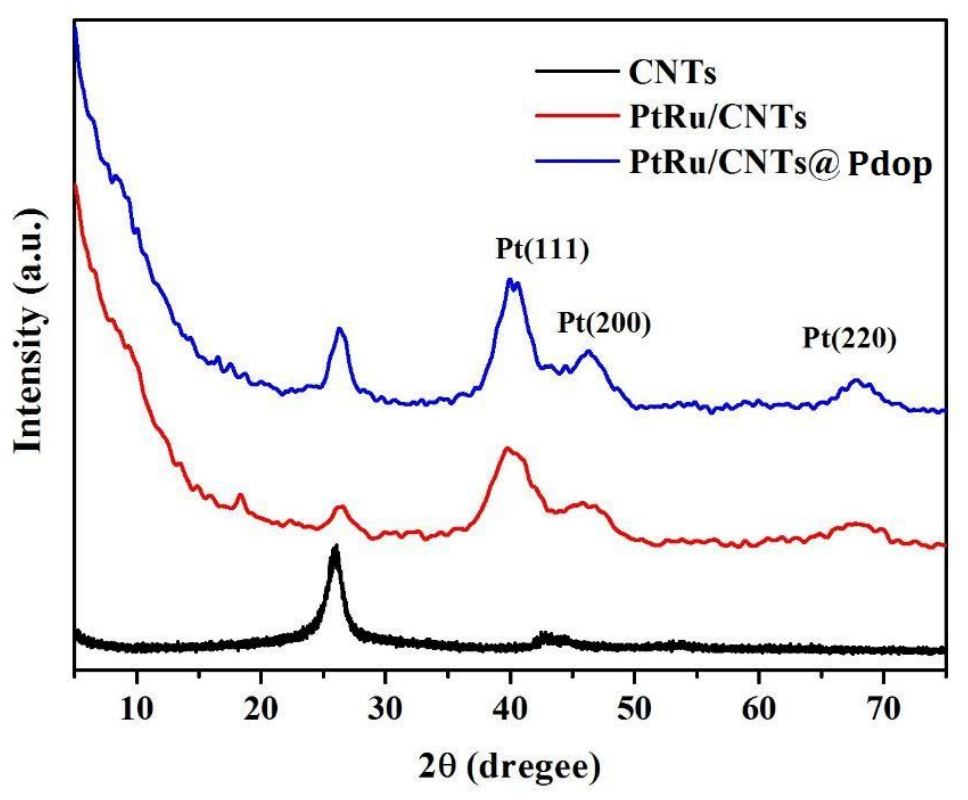

Figure 4. 

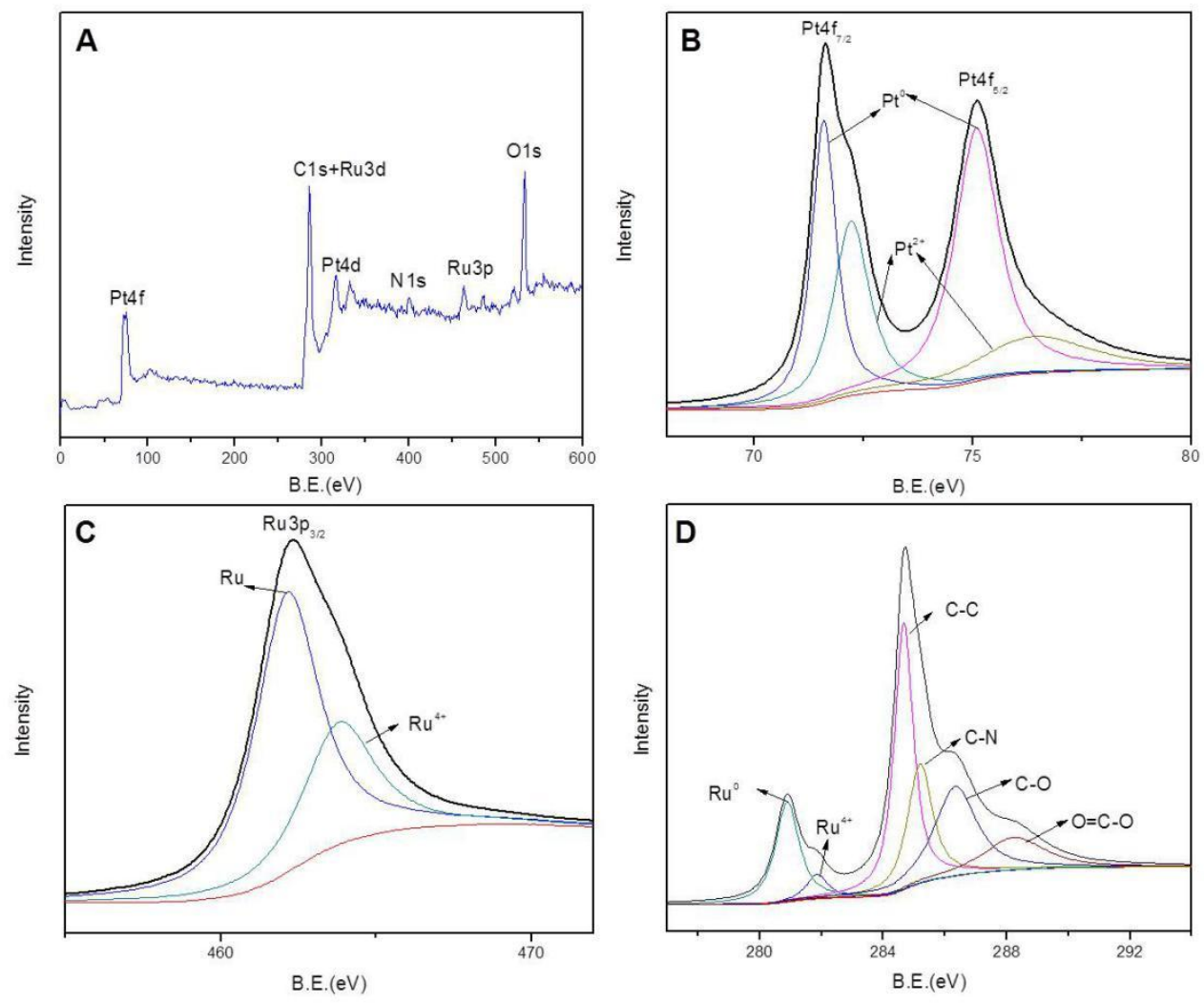

Figure 5 

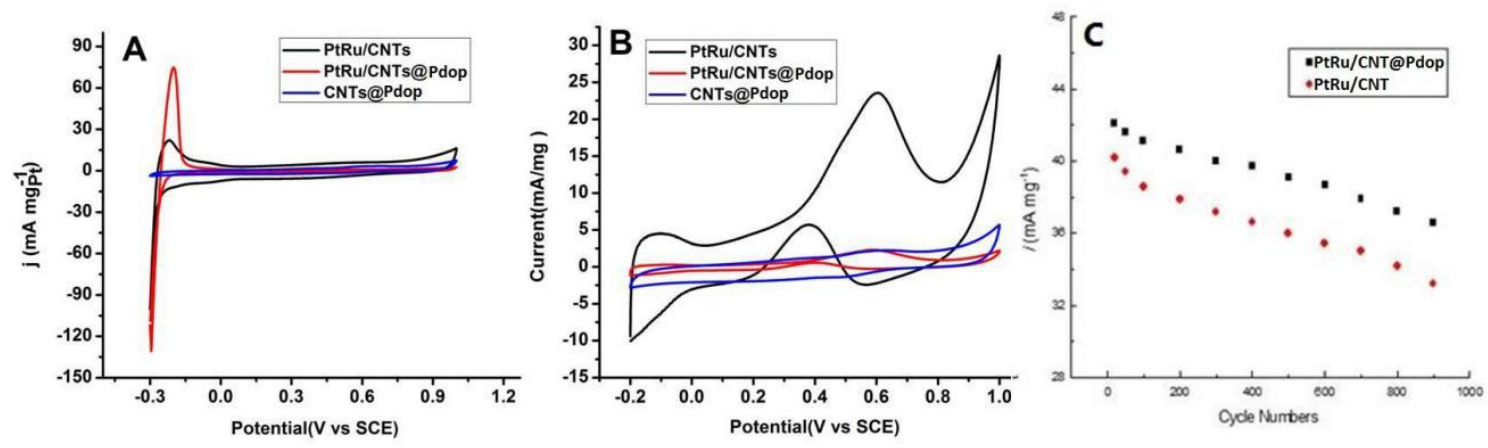

Figure 6. 
Facile and one-pot synthesis of uniform PtRu nanoparticles on polydopamine-modified multiwalled carbon nanotubes for direct methanol fuel cell application

Fengxia Chen, ${ }^{\text {a,b }}$ Junkai Ren, ${ }^{a}$ Qian He, ${ }^{a}$ Jun Liu, ${ }^{c^{*}}$

$$
\text { Rui Song }{ }^{\mathrm{a}^{*}}
$$

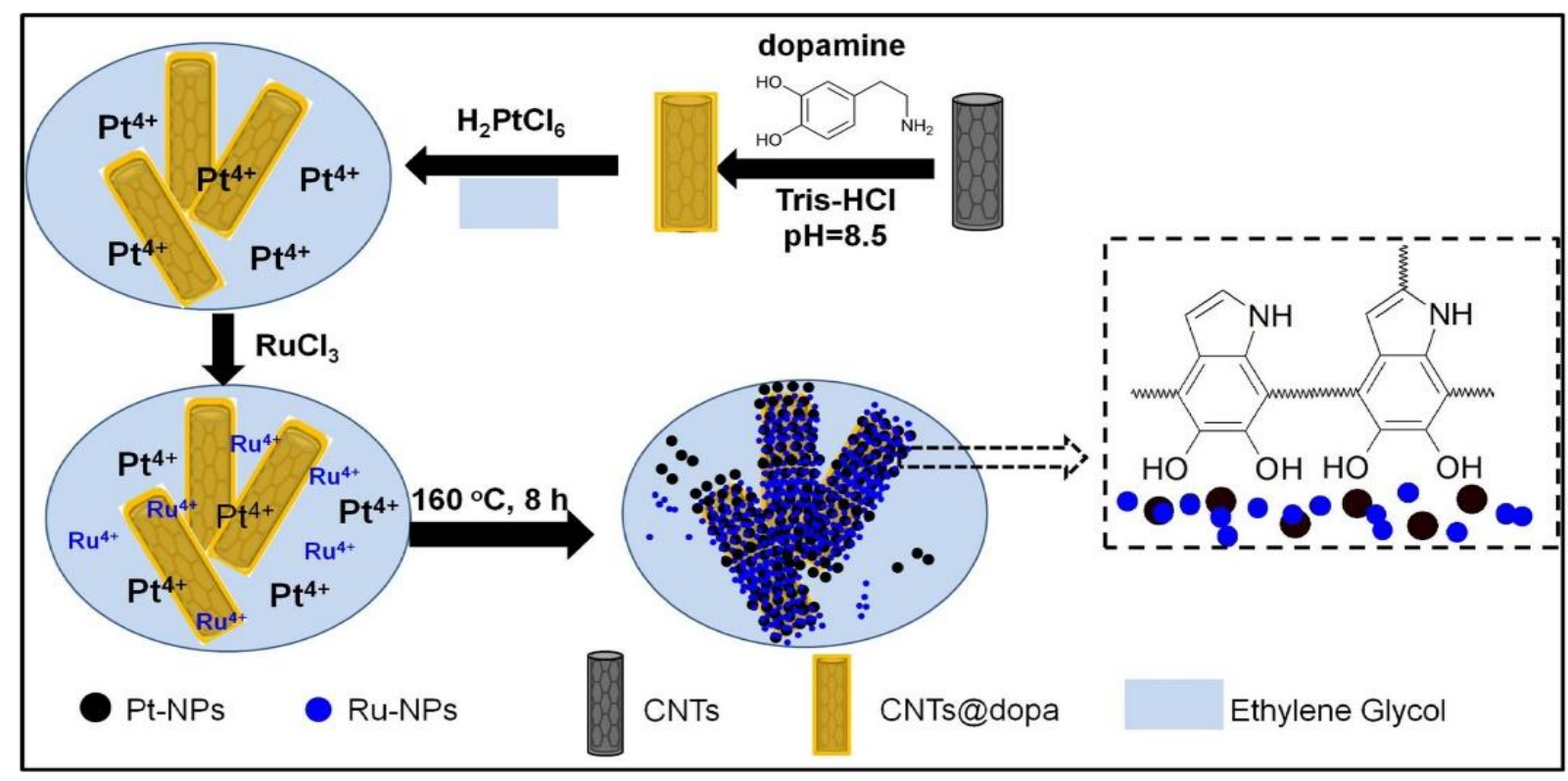

PtRu NPs immobilized on the Pdop coating-modified MWCNTs bimetallic alloy catalysis used as the direct methanol fuel cell application. 